\title{
EEG ACTIVITY DURING BALANCE PLATFORM TEST IN HUMANS
}

\author{
Vladimir Khorev, Artem Badarin \\ Neuroscience and Cognitive \\ Technology Lab \\ Center for technologies in robotics and \\ mechatronics components \\ Innopolis University \\ Russia \\ v.horev@innopolis.ru, a.badarin@innopolis.ru
}

\author{
Vladimir Antipov \\ Center for technologies in robotics and \\ mechatronics components \\ Innopolis University \\ Russia \\ v.antipov@innopolis.ru
}

\author{
Vladimir Maksimenko, Semen Kurkin \\ Neuroscience and Cognitive \\ Technology Lab \\ Center for technologies in robotics and \\ mechatronics components \\ Innopolis University \\ Russia \\ v.maksimenko@innopolis.ru, s.kurkin@innopolis.ru
}

Article history:

Received 14.10.2019, Accepted 26.11.2019

\begin{abstract}
In order to analyze different human brain states related to perception and maintaining of body posture, we implemented an experiment with a balance platform. It is known the cerebral cortex regulates subcortical postural centers to maintain upright balance and posture and balance demands. However, the cortical mechanisms that support standing balance remain elusive. In this work, we present an EEG-based analysis during execution of balance responses with distinct postural demands. The results suggest the existence of common features in the EEG structure associated with distinct activity during balance maintaining. This may give new directions for future research in the field of brain activity, and for the development of brain-computer interfaces.
\end{abstract}

\section{Key words}

EMG, EEG, balance, activity, posture

\section{Introduction}

Reactions to maintain dynamic balance and stability of the body posture play an important role in everyday life, allowing a person to avoid injuries and, at the same time, save energy spent on unnecessary actions. Maintaining the vertical position of the body is a complex problem of integrated control [Dutt-Mazumder, 2018].
The cerebral cortex and central nervous system play integral roles in postural control, incorporating information from visual, somatosensory, and vestibular systems to carry out the corrective motions needed to maintain balance [Takusaki, 2017]. The role of the central nervous system and subcortical structures for the innate generation of feedforward and feedback adaptive adjustments to reduce the risk of balance loss is well documented in literature [Edmunds, 2019; Bell, 2012; Luu, 2012].

Some studies have found an interaction between postural control and cognitive task performance, indicating that postural control is not a fully automatic process but rather may require active cognitive processes [Yogev, 2008], including complex information processing [Maksimenko, 2018], such as perception, decisionmaking and motor control [Chen, 2018; Cholak, 2019]. While many tools have been employed to study neurocognitive processes in this regard, the use of electroencephalography (EEG) as a neuroimaging technique remains disparately reported. Nonetheless, extant literature has investigated the utility of EEG through the examination of evoked potentials associated with balance perturbation [Hulsdunker, 2015; Solis-Escalante, 2019]. The mechanical aspects of this problem have been studied well and even implemented in practical applications in robotic solutions [Collins, 2005; Latella, 2019] quite well. However, the simultaneous whole-body estimation 


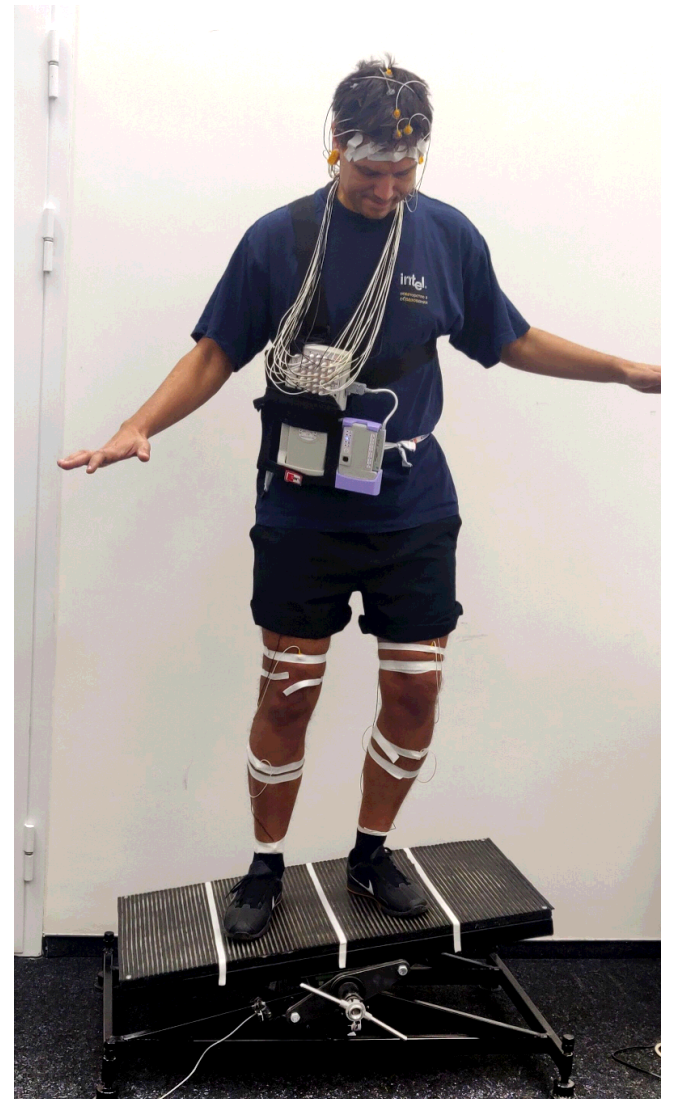

Figure 1. A photo of the experimental setup.

of the human kinematics and dynamics, such as joint torques and internal forces for controlling the movement, mechanisms that include processing information in the higher nervous system in response to non-standard stimuli (respectively, excluding fast spinal reflexes to typical perturbations) are not well understood and leave areas for further research. Moreover, most of the experimental works use a one-side approach when subjects only passively react to the balance perturbation without interaction [Adkin, 2008; Rodrick, 2013; Mierau, 2017]. That means no effective learning and feedback essential for performance improvement [Hiroaki, 2018].

\section{Results}

\section{Experimental Setup}

A series of experimental works in a group of 7 unpaid conditionally healthy male volunteers was carried out. Age of the volunteers ranged from 25 to 36 years, the physical conditions corresponded to the characteristics of a normal body mass index and an average level of physical activity. All volunteers were instructed before conducting the research to observe the regime of full night rest for three days. Studies were conducted in the morning and afternoon periods (9 AM - 1 PM) 2 hours after a healthy meal with limited consumption caffeine and (or) other stimulating additives to food.
The duration of the experiment was 50 minutes. During the recording of signals, subjects were standing on the balance platform (Fig. 1). The structure of the experiment included three 10-minutes sessions with two 5 -minutes rest pauses between them. Pre-registration of background (BG) activity without subject performing special instructions was carried out for 3 minutes.

All of the subjects were instructed to maintain balanced posture during their attempts. We especially note the fact that no volunteers had the opportunity to train their ability to maintain balance before the experiment and, thus, the study was conducted with untrained operators. This is important because recent studies have shown that trained subjects (such as athletes) have dif-

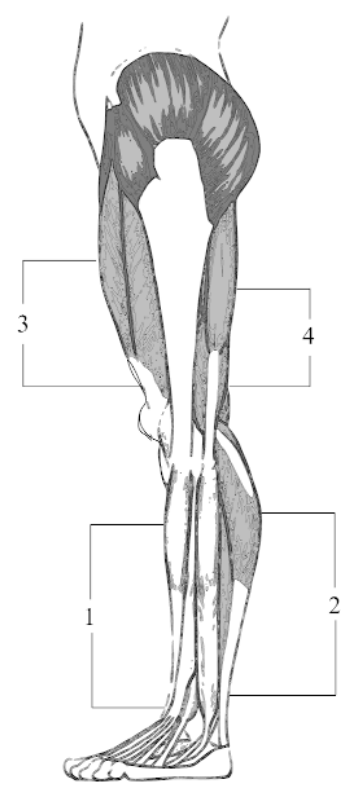

Figure 2. Schematic representation of the subject leg with 4 connected electrodes for measuring EMG signals during the experiment.

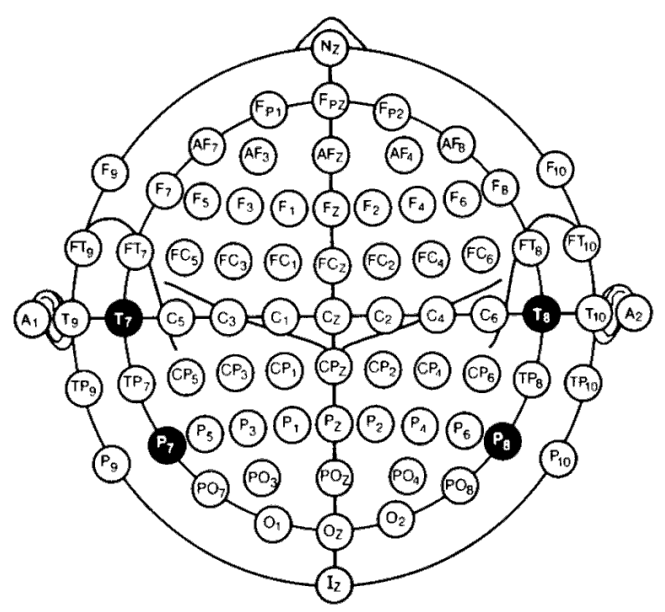

Figure 3. The international 10-10 system of EEG electrodes arrangement. 


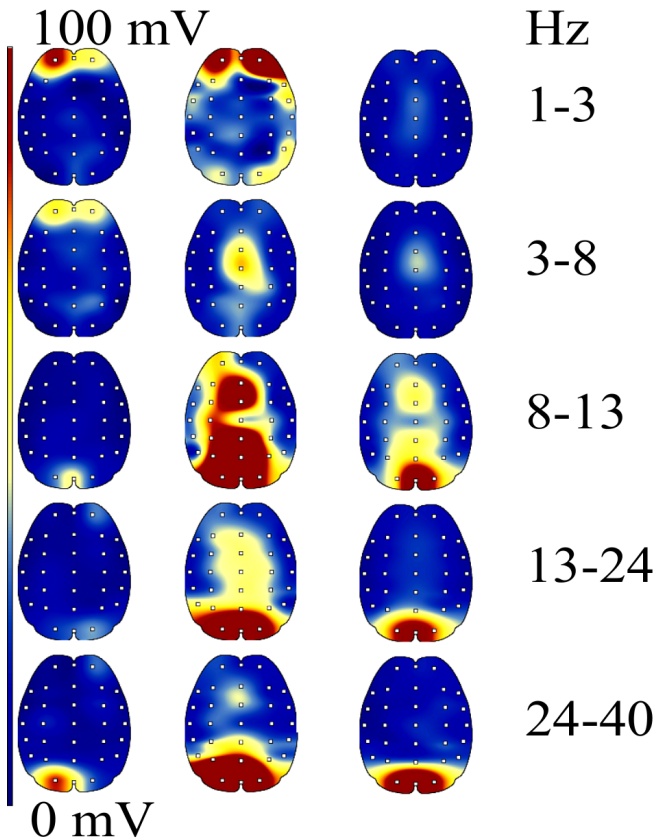

Figure 4. Topoplots of the power spectral density distribution over the head during the background activity registration (left column), active movement (middle) and maintaining balanced posture (right) for one of the subjects.

ferent spectral activity distribution in comparison with untrained. Specifically, lower reduction in amplitude of EEG oscillations at dominant alpha rhythms were observed during different posture keeping experiments. This may support "neural efficiency" hypothesis in sense of a more selective involvement of related cortical areas [Del Percio, 2009; Guo, 2017].

During the postural session, platform angle, platform speed, electromyography (EMG), Microsoft Kinect sensor signal, and 32-channels EEG data were recorded continuously according to the standard "10-10" configuration (see the scheme of the EEG electrodes arrangement in Fig. 3). As shown on the scheme of the EMG electrodes in Fig. 2), arrangement included next muscles: Tibialis Anterior (1), Gastrocnemius (2), Rectus Femoris (3), Semitendinosus (4). Registration of EEG signals was carried out using a multichannel electroencephalograph-analyzer "Encephalan131-03", model 10 (Taganrog, Russian Federation) with a set of standard sensors. Signals were recorded at a sampling frequency of $250 \mathrm{~Hz}$ with a 12-bit resolution. Data frequency ranged from $0.016 \mathrm{up}$ to $70 \mathrm{~Hz}$ using a notch filter on $50 \mathrm{~Hz}$. Variations of spectral power were calculated to assess whether changes in postural control and balance keeping were significantly different. To do this, power spectral density values were extracted for all EEG electrodes and averaged within the following frequency bands: delta $(1-3 \mathrm{~Hz})$, theta $(3-7 \mathrm{~Hz})$, alpha $(8-13 \mathrm{~Hz})$, beta $(14-24 \mathrm{~Hz})$ and gamma $(24-40 \mathrm{~Hz})$. These dependencies are presented in Fig. 4. Although the EMG and Kinect signals were used as additional balance and for the body movement activity monitoring in this study, they would help us in a future studies. For the first step we are planning to determine the level at which the cortical activity is involved in muscle synergy or individual muscle level. The next step would include establishing mechanics behind muscle synergy and if possible implement it in autonomous robotic solution.

\section{Results}

EEG activity clearly shows the difference in patterns corresponding to motor activity as compared with the background passive regime. The spectral structure of trials corresponding to the events associated with balance motor activity varies significantly in intensity of oscillations on various recording channels. A more detailed time-frequency analysis is required in terms of the structure of the specific features in EEG data associated with the preparation and maintaining the motor activity of a particular type. During the active balance search, most of the energy is focused in the sensory cortex, predominantly in the alpha frequency band. Also worth mentioning low-frequency activity in the front cortex presumably related to the cognitive activity during the task (thinking of the best tactics to get the balance).

During the balance keeping, frontal cortex activity decreases and only necessary sensorimotor centers remain active. High-frequency band activity in the occipital lobe can be a result of both unusual visual cortex stimulation and neck muscle activity. Sources in or near anterior cingulate, anterior parietal, superior dorsolateral prefrontal and medial sensorimotor cortex exhibited significantly larger mean spectral power in the alpha $(8-13 \mathrm{~Hz})$ and beta bands $(14-24 \mathrm{~Hz})$ during active balance search compared to the balance keeping and background.

Comparative analysis of platform angle data, EMG and Kinect data allowed to determine the moments of balance keeping during the experimental sessions. The EMG activity helped to understand the periods of active and more relaxed balance keeping. The Kinect signal variance was used as an additional factor of learning, as it tends to decrase from session to seeion. The balance was registered if the angle of the platform was within \pm 5 -degree range, angle velocity of the platform was within $\pm 0.03 \mathrm{rad} / \mathrm{s}$ for more than $1 \mathrm{~s}$ time period. The distributions of the balance keeping times $\Delta t$ (see Fig. 5) during the sessions demonstrate the tendency to increase for all the subjects that is most likely connected to the learning process of the subjects. The widening in the last part of the third session caused by the fatigue as subjects verbally noted after the experiments.

The angle distribution can be an interesting characteristic by itself as it shows the progress in the operator learning between the experimental sessions. As shown in Fig. 6, the distribution with border peaks of the first session means very low and random balance moments when the platform was leaning on one of the sides most of 


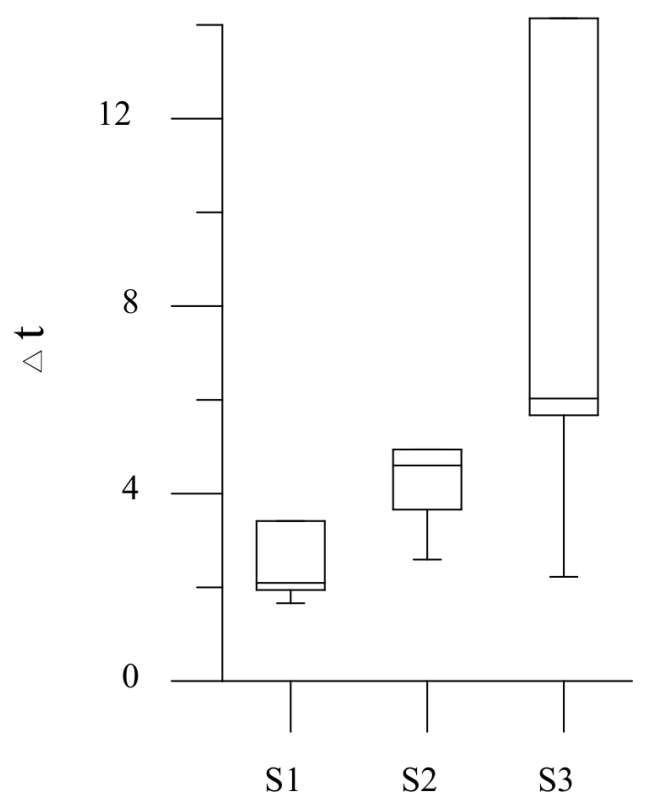

Figure 5. The distributions of balance keeping times $\Delta \mathrm{t}$ during the sessions $S_{i}$ with their respective standard deviations and quartile ranges.

the time. The distribution of the second session demonstrates a slight increase in the center with a slight decline in the side peaks. Finally, in the third session, distribution has a maximum value near zero degrees angle what corresponds to the nearly horizontal position of the platform that was the most comfortable balance position for the subject.

\section{Conclusion}

Thus, the decrease in spectral power in the alpha (8$13 \mathrm{~Hz})$ and beta bands $(14-40 \mathrm{~Hz})$ was observed in the sensorimotor cortex during active balance search compared to the balance keeping and background. That could possibly mean that the task of postural control demands activation of the task-related brain activity in frontal-parietal areas during active balance seeking but not so much during balance keeping. This study adds to a newly evolving understanding of the role of cortical processing in the control of postural balance, which may have an important impact on future studies that examine interactions between cortical activity and postural control.

\section{Acknowledgements}

The research is supported by grant of the Russian Science Foundation (project No. 17-72-30003).

\section{References}

Adkin A. L., Campbell A. D., Chua R., Carpenter, M. G. (2008). The influence of postural threat on the cortical response to unpredictable and predictable postural perturbations. In Neuroscience Letters, 435, pp. 120-125.

Bell, M. A., Cuevas, K. (2012). Using EEG to study cognitive development: issues and practices. In Journal of cognition and development, 13(3), pp. 281-294. Chen, Y., Yu Y., Niu R. and Liu Y. (2018). Selective Effects of Postural Control on Spatial vs. Nonspatial

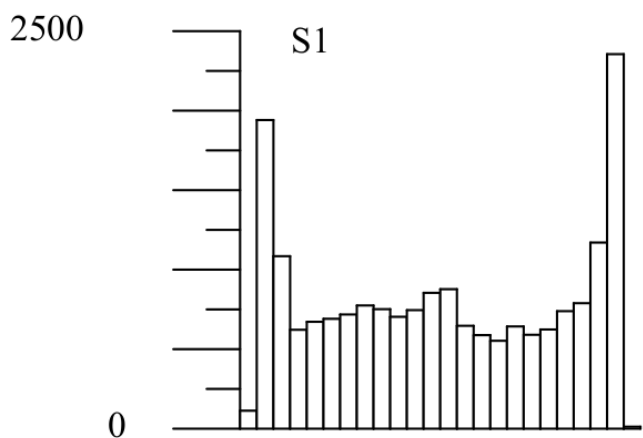

2500

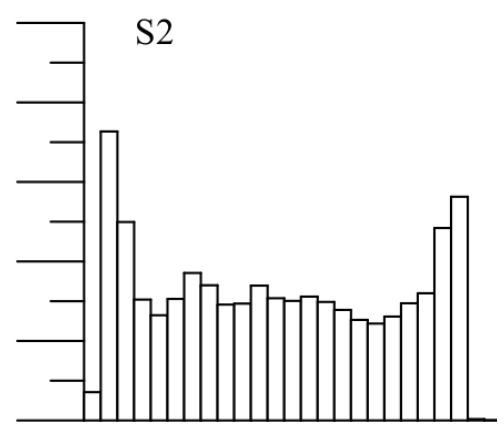

2500

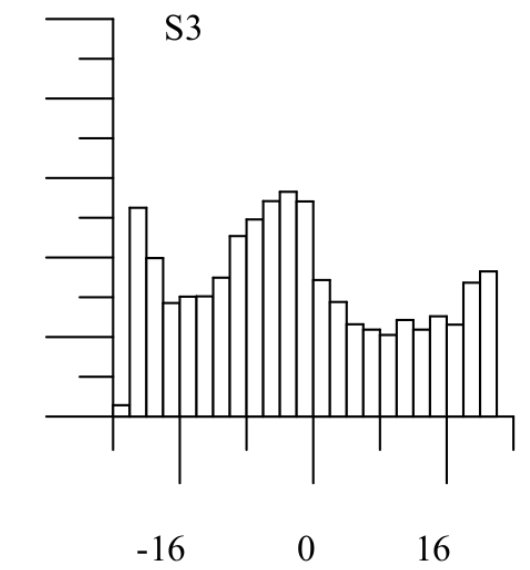

Angle, degrees

Figure 6. The distribution of platform angle values during the sessions for one of the subjects. 
Working Memory: A Functional Near-Infrared Spectral Imaging Study. In Frontiers in Human Neuroscience, (12), pp. 243.

Chholak, P., Niso, G., Maksimenko, V. A, Kurkin, S. A., Frolov, N. S., Pitsik, E. N., Hramov, A. E., Pisarchik, A. N. (2019). Visual and kinesthetic modes affect motor imagery classification in untrained subjects. In Scientific reports, 9(1), pp. 9838.

Collins, S., Ruina, A., Tedrake R., Wisse, M. (2005). Efficient bipedal robots based on passive-dynamic walkers. In Science, 307(5712), pp. 1082-1085.

Del Percio, C., Babiloni, C., Marzano, N., Iacoboni, M., Infarinato, F., Vecchio. F., Lizio, R., Aschieri, P., Fiore, A., Torán, G., Gallamini, M., Baratto, M., Eusebi, F. (2009). "Neural efficiency" of athletes brain for upright standing: a high-resolution EEG study. In Brain Research Bulletin, 79(3-4), pp. 193-200.

Dutt-Mazumder, A., Dhar, S., Dutt-Mazumder, C. (2018). Postural Stability Variables for Dynamic Equilibrium. In Journal of Nature and Science, 4(12), e541.

Edmunds, K., Petersen, H., Hassan, M., Yassine, S., Olivieri, A., Barollo, F., Friðriksdóttir, R., Edmunds, P., Gislason, M. K., Fratini, A., Gargiulo, P. (2019). Cortical recruitment and functional dynamics in postural control adaptation and habituation during vibratory proprioceptive stimulation. In Journal of Neural Engineering, 16(2), pp. 026037.

Guo, Z., Li, A., Yu, L. (2017). "Efficiency" of Athletes Brain during Visuo-Spatial Task: An fMRI Study on Table Tennis Players. In Frontiers in Behavioral Neuroscience, 11, art 72 .

Hiroaki, M., Takahiro, H., Yuya, M., Foti, D., Hajcak, G. (2018). Feedback-Related Electroencephalogram Oscillations of Athletes With High and Low Sports Anxiety. In Frontiers in Psychology, 9, pp. 1420.

Hülsdünker, T., Mierau, A., Neeb, C., Kleinöder, H., Strüder, H. K. (2015). Cortical processes associated with continuous balance control as revealed by EEG spectral power. In Neuroscience Letters, 592, pp. 1-5. Latella, C., Traversaro, S., Ferigo, D., Tirupachuri, Y., Rapetti, L., Andrade Chavez, F. J., Nori, F., Pucci, D. (2019). Simultaneous Floating-Base Estimation of Human Kinematics and Joint Torques. In Sensors (Basel), 19(12), 2794.

Luu, B. L., Inglis J.T., Huryn T.P., Van der Loos, H. F., Croft, E. A., Blouin, J. S. (2012). Human standing is modified by an unconscious integration of congruent sensory and motor signals. In The Journal of Physiology, 590(22), pp. 5783-5794.

Maksimenko, V. A., Hramov, A. E., Grubov, V. V., Nedaivozov, V. O., Makarov, V. V., Pisarchik, A. N. (2018). Nonlinear effect of biological feedback on brain attentional state. In Nonlinear Dynamics, 95(3), pp. 1923-1939.

Mierau, A., Pester, B., Hülsdünker, T., Schiecke, K., Strüder, H. K., Witte, H. (2017). Cortical correlates of human balance control. In Brain Topography, 30(4), pp. 434-446.

Rodrick, D., Jayaprakash, V. (2013). Neural Mechanisms of Anticipatory Balance Control. In Proceedings of the Human Factors and Ergonomics Society Annual Meeting, 57(1), pp. 915-919.

Solis-Escalante, T., van der Cruijsen, J., de Kam, D., van Kordelaar, J., Weerdesteyn, V., Schouten, A. C. (2019). Cortical dynamics during preparation and execution of reactive balance responses with distinct postural demands. In Neuroimage, 188, pp. 557-571.

Takakusaki, K. (2017). Functional Neuroanatomy for Posture and Gait Control. In Journal of movement disorders, 10(1), pp. 1-17.

Yogev, G., Hausdorff, J. M., Giladi, N. (2008). The role of executive function and attention in gait. In Movement Disorders, 23(3), pp. 329-342. 